The TAIGA experiment: from cosmic ray to gamma-ray astronomy in the Tunka valley

This content has been downloaded from IOPscience. Please scroll down to see the full text.

2016 J. Phys.: Conf. Ser. 718052006

(http://iopscience.iop.org/1742-6596/718/5/052006)

View the table of contents for this issue, or go to the journal homepage for more

Download details:

IP Address: 129.13.72.197

This content was downloaded on 09/08/2017 at 07:34

Please note that terms and conditions apply.

You may also be interested in:

The Tunka Radio Extension: Latest Analysis Results

D Kostunin, P A Bezyazeekov, N M Budnev et al.

The Tunka detector complex: from cosmic-ray to gamma-ray astronomy

N Budnev, I Astapov, N Barbashina et al.

The Tunka-Grande experiment

R.D. Monkhoev, N.M. Budnev, A. Chiavassa et al.

TAIGA the Tunka Advanced Instrument for cosmic ray physics and Gamma Astronomy — present status and perspectives.

N M Budnev, I I Astapov, A G Bogdanov et al.

The HiSCORE experiment and its potential for gamma-ray astronomy

M Tluczykont, D Hampf, U Einhaus et al.

Tunka-Rex: a Radio Extension of the Tunka Experiment

F G Schröder, D Besson, N M Budnev et al.

THE RESULTS OF PHOTOMETRIC RECORDING OF THE OCCULTATION OF THE STAR HIP 97157 BY QAPERREIDI(4H) THE TELESCOPE OF THE GLOBAL MASTER ROBOTIC NET

E. M. Trunkovsky, E. S. Gorbovskoy, D. V. Denisenko et al.

First deployment and prototype data of HiSCORE

R Nachtigall, M Kunnas, S N Epimakhov et al.

Problem of the knee in the cosmic ray energy spectrum

N N Kalmykov 
XIV International Conference on Topics in Astroparticle and Underground Physics (TAUP 2015) IOP Publishing Journal of Physics: Conference Series 718 (2016) 052006

\title{
The TAIGA experiment: from cosmic ray to gamma-ray astronomy in the Tunka valley
}

\author{
N Budnev ${ }^{2}$, I Astapov', P Bezyazeekov ${ }^{2}$, A Bogdanov $^{9}$, V Boreyko ${ }^{10}$, M Büker $^{6}, \mathrm{M}$ \\ Brückner $^{11}$, A Chiavassa ${ }^{4}, O$ Chvalaev $^{2}, O_{\text {Gress }}{ }^{2}$, T Gress $^{2}, O$ Grishin $^{2}, A$ \\ Dyachok $^{2}$, S Epimakhov ${ }^{6}$, O Fedorov ${ }^{2}$, A Gafarov $^{2}, \mathrm{~N}_{\text {Gorbunov }}{ }^{10}, \mathrm{~V}$ \\ Grebenyuk $^{10}$, A Grinuk ${ }^{10}$, A Haungs ${ }^{12}$, R Hiller ${ }^{12}$, D Horns ${ }^{6}$, T Huege ${ }^{1}$, A \\ Ivanova $^{2}$, A Kalinin ${ }^{10}$, N Karpov ${ }^{1}$, N Kalmykov ${ }^{1}$, Y Kazarina ${ }^{2}$, N Kirichkov ${ }^{2}$, S \\ Kiryuhin $^{2}$, M Kleifges ${ }^{13}$, R Kokoulin ${ }^{~}$, K Komponiest ${ }^{9}$, A Konstantinov ${ }^{1}$, E \\ Korosteleva $^{1}$, D Kostunin ${ }^{12,2}$, V Kozhin ${ }^{1}$, O Krömer ${ }^{\mathrm{m}}$, M Kunnas ${ }^{6}$, L \\ Kuzmichev $^{1,2}$, V Lenok ${ }^{2}$, B Lubsandorzhiev ${ }^{3}$, N Lubsandorzhiev ${ }^{1}$, R Mirgazov ${ }^{2}$, \\ R Mirzoyan ${ }^{5,2}, \mathbf{R}$ Monkhoev $^{2}$, R Nachtigall $^{6}$, A Pakhorukov ${ }^{2}$, M Panasyuk $^{1}, \mathrm{~L}$ \\ Pankov $^{2}$, A Perevalov ${ }^{2}$, A Petrukhin', V Platonov ${ }^{2}$, V Poleschuk ${ }^{2}$, E Popova $^{1}$, A

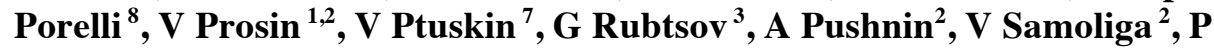 \\ Satunin $^{7}$, F Schröder ${ }^{12}$, Yu Semeney ${ }^{2}$, A Silaev ${ }^{1}$, A Silaev (junior) ${ }^{1}$, A \\ Skurikhin $^{1}$, V Slucka $^{10}$, C Spiering $^{8}$, L Sveshnikova $^{1,2}$, V Tabolenko ${ }^{2}$, B \\ Tarashansky ${ }^{2}$, A Tkachenko ${ }^{10}, L_{\text {Tkachev }}{ }^{10}, M$ Tluczykont ${ }^{6}$, D Voronin ${ }^{2}, R$ \\ Wischnewski $^{8}$, A Zagorodnikov ${ }^{2}$, V Zurbanov ${ }^{2}$, I Yashin' \\ ${ }^{1}$ Skobeltsyn Institute of Nuclear Physics MSU, Moscow, Russia \\ ${ }^{2}$ Institute of Applied Physics, ISU, Irkutsk, Russia \\ ${ }^{3}$ Institute for Nuclear Research of RAN, Moscow, Russia \\ ${ }^{4}$ Dipartimento di Fisica Generale Universiteta di Torino and INFN, Torino, Italy \\ ${ }^{5}$ Max-Planck-Institute for Physics, Munich, Germany \\ ${ }^{6}$ Institut für Experimentalphysik, University of Hamburg, Germany \\ ${ }^{7}$ IZMIRAN, Troitsk, Moscow Region, Russia \\ ${ }^{8}$ DESY, Zeuthen, Germany \\ ${ }^{9}$ NRNU MEPhI, Moscow,Russia \\ ${ }^{10}$ JINR, Dubna, Russia \\ ${ }^{11}$ Institute for Computer Science, Humboldt-University, Berlin, Germany \\ ${ }^{12}$ Institut für Kernphysik, KIT, Karlsruhe, Germany \\ ${ }^{13}$ Institut für Prozessdatenverarbeitung und Elektronik, KIT, Karlsruhe, Germany
}

E-mail: nbudnev@api.isu.ru

\begin{abstract}
The physical motivations and advantages of the new gamma-observatory TAIGA (Tunka Advanced Instrument for cosmic ray physics and Gamma Astronomy) is presented. The TAIGA array is a complex, hybrid detector for ground-based gamma-ray astronomy for energies from a few $\mathrm{TeV}$ to several $\mathrm{PeV}$ as well as for cosmic ray studies from $100 \mathrm{TeV}$ to several EeV. The TAIGA will include the wide angle Cherenkov array TAIGA-HiSCORE with $\sim 5 \mathrm{~km}^{2}$ area, a net of 16 IACT telescopes (with FOV of about 10x10 degree), muon detectors with a total area of up to $2000-3000 \mathrm{~m}^{2}$ and the radio array Tunka-Rex.
\end{abstract}


XIV International Conference on Topics in Astroparticle and Underground Physics (TAUP 2015) IOP Publishing Journal of Physics: Conference Series 718 (2016) $052006 \quad$ doi:10.1088/1742-6596/718/5/052006

\section{Introduction}

In recent years gamma-ray astronomy became the most dynamically developing field of astroparticle physics. More than 150 sources of $\mathrm{TeV}$ gamma - ray were discovered and studied. However, until now there was not detected a single photon with energy above $80 \mathrm{TeV}$. Essentially, this is explained due to the fact that the most of formerly and currently existing arrays are a network of Imaging Atmospheric Cherenkov Telescopes (IACT): HEGRA, H.E.S.S., MAGIC and VERITAS with the level of sensitivity optimized for the energy range $100 \mathrm{GeV}-20 \mathrm{TeV}$. Although, there are a lot of fundamental questions without answers about processes in the range of high and ultra-high energies. The so-called $4^{\text {th }}$ generation IACT array CTA as well HAWC and LHAASO will have substantially higher sensitivity than the existing telescopes in the energy range of up to $100 \mathrm{TeV}$, but not sufficient to solve all problems of very high energy gamma - astronomy.

To solve many crucial tasks of high energy gamma-astronomy as well cosmic ray physics we propose to design and to construct the gamma-observatory TAIGA (Tunka Advanced Instrument for cosmic ray physics and Gamma Astronomy) - a complex hybrid detector system including: wide-angle Cherenkov detectors, IACTs, electron, muon and radio detectors grouped into 6 arrays. Common operation of an array of IACTs and a wide-angle Cherenkov array can allow one to abstain from simultaneous measurement of the EAS in stereoscopic mode by several closely packed IACT and thus allows to increase the distance between IACT up to $600-1000 \mathrm{~m}$ reducing the number of telescopes needed by a factor of four times or more. In the combination of both techniques, the timing array provides the information about shower core position, incidence angle and energy, and the IACT images are used for the identification of the primary particle.

TAIGA will be located in the Tunka valley, about $50 \mathrm{~km}$ from Lake Baikal in Siberia, Russia, were since 2009 the full-size Tunka-133 Cherenkov EAS detector is in operation [1]. The experience of Tunka-133 on the energy spectrum and mass composition of cosmic rays, together with it's existing infrastructure are important factors for selecting this location. TAIGA will combine six arrays with different types of detectors.

\section{The Tunka-133 array}

The Tunka-133 array consists of 175 wide-angle Cherenkov detectors distributed over $3 \mathrm{~km}^{2}$ area $[1,2]$. The detectors are grouped into 25 clusters, each with 7 detectors - six hexagonally arranged detectors and one in the center. The distance between the detectors in each cluster is $85 \mathrm{~m}$.

The energy spectrum and mass composition of cosmic rays in the range of 6-1000 PeV has been reconstructed using data from 5 winter seasons of measurements with the Tunka-133 array [2]. The spectrum has a rather complicated structure with different power-law index and points out reliably the existence of a "second knee" at $300 \mathrm{PeV}$, probably, it is manifestation of a transition from galactic to extragalactic origin of the cosmic rays. We find a decrease of the mean (in logarithm) of the atomic number or composition, this lightening at energies higher than $100 \mathrm{PeV}$ also points to a transition to extragalactic cosmic rays [3].

\section{The TAIGA-HiSCORE array}

The principle of the TAIGA-HiSCORE array follows the idea outlined in [4,5]. It is rather similar to the one used for the Tunka-133 array. Again, this method is based on the sampling of the Cherenkov light front of air showers. The TAIGA-HiSCORE will consist of an array of 500 wide-angle (field of view $0,6 \mathrm{sr}$ ) light-sensitive detector stations, distributed with spacing $75-200 \mathrm{~m}$ over an area of a five square kilometre, probably the array area will be extended in future. The array threshold for gammaray induced EAS is $30 \mathrm{TeV}$, for cosmic rays $100 \mathrm{TeV}$. The TAIGA -HiSCORE data allow to reconstruct the arrival direction of the EAS with an accuracy of about 0.1 degree, the core location to 5-6 m, energy to $10-15 \%$ and shower maximum height $X_{\max } 20-25 \mathrm{~g} / \mathrm{cm}^{2}$.

A TAIGA-HiSCORE [6,7] detector stations consist of two boxes (figure 1). The first is the Cherenkov box with four PMTs, each equipped with a light-collecting Winston cone of $30^{\circ}$ halfopening angle pointing to the zenith. 


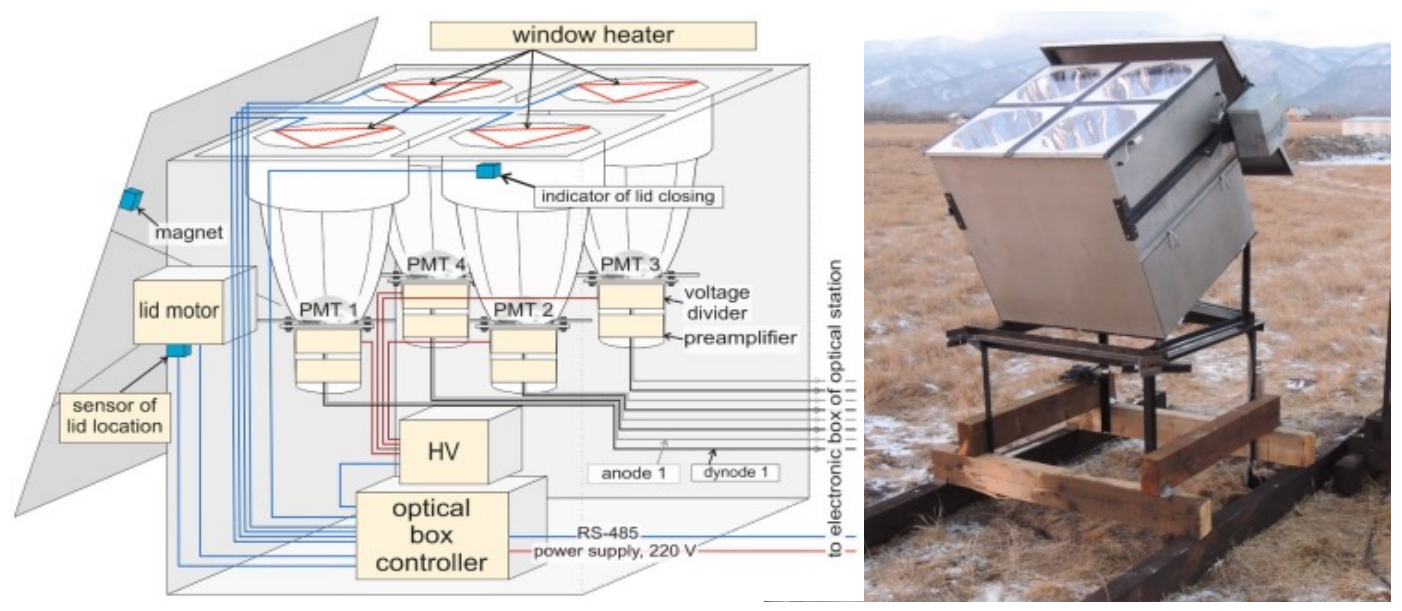

Figure 1. Schematic view (left) and outer view (right) of the TAIGA-HiSCORE optical box.

The data acquisition system (DAQ), the slow control for the PMT-high voltage, environmental control and some auxiliary electronics (figure 2) are placed in a special temperature-controlled box. Fast signal read-out and digitization in the $\mathrm{GHz}$ regime is provided by a read-out board with up to 8 channels based on the Domino Ring Sampler DRS 4 chip, with a depth of 1024 cells at a resolution of up to $0.5 \mathrm{~ns}$ per cell $(2 \mathrm{GHz})$. To synchronize all stations in the array to sub-nsec precision we use a hybrid approach, combining a custom-made synchronization technique (100 MHz clocks distributed over separate fibers from the array center), and the new White Rabbit Ethernet-based technology (with synchronization and Gbit data traffic over a single fiber) [13].

The configuration of the Tunka-HiSCORE setup of $2015 \mathrm{y}$ is shown in figure 3 . It comprises 28 detectors at $106 \mathrm{~m}$ spacing forming a super-cell structure. The total area of the setup is $0.25 \mathrm{~km}^{2}$. To increase the observation time for the gamma-ray source in the Crab Nebula, all optical boxes are tilted towards South by 25 degrees.
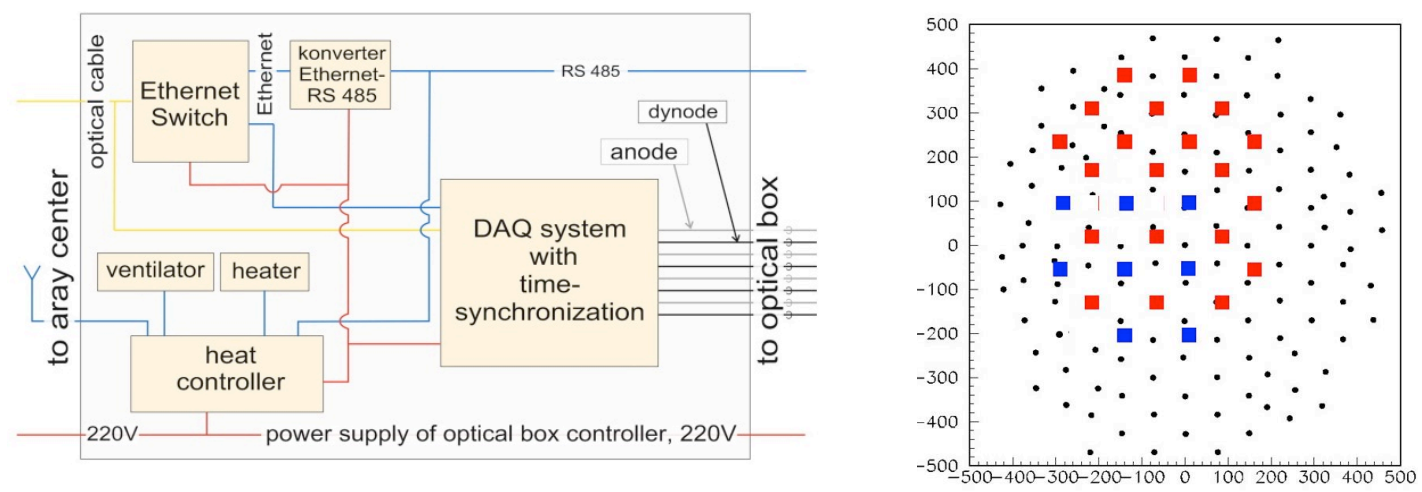

Figure 2. Layout of the TAIGA-HiSCORE electronics box .
Figure 3. Sketch of the layout of the TAIGAHiSCORE array (blue and red) and the inner Tunka-133 stations (black dots).

\section{The TAIGA-IACT array}

The TAIGA- IACT array will include 16 Imaging Atmospheric Cherenkov Telescopes distributed with $600-1000 \mathrm{~m}$ spacing over an area of $5 \mathrm{~km}^{2}$. Each HEGRA-like telescope of the array will be composed of a mosaic, 34-segment reflector in Davis-Cotton design, with a diameter of individual mirrors $60 \mathrm{~cm}$. The full diameter of the reflector is $4.3 \mathrm{~m}$, the area $\sim 10 \mathrm{~m}^{2}$, the focal length $4.75 \mathrm{~m}$. The Imaging camera consist of 547 photomultipliers and will have a field of view (FOV) of 9,72 $\mathrm{x}$ 9,72 degrees and an angular size of 0.36 degrees per pixel. The entire array of pixels is divided into 
XIV International Conference on Topics in Astroparticle and Underground Physics (TAUP 2015) IOP Publishing Journal of Physics: Conference Series 718 (2016) $052006 \quad$ doi:10.1088/1742-6596/718/5/052006

clusters of 28 PMTs each. Each cluster includes an electronic board MAROC, the basic element of which is a 64-channel chip ASIC MAROC3.

The IACT array energy threshold for gamma-rays is $1 \mathrm{TeV}$, the sensitivity in the energy range 1-20 $\mathrm{TeV}$ is $10^{-12} \mathrm{erg} \mathrm{cm}^{-2} \mathrm{~s}^{-1}$ for 50 hours of observation with angular of resolution 0.03 degree. The reconstruction of core position, arrival direction and energy of coincident EAS events by the TAIGAHiSCORE array will help the IACTs to reject the high energy background events on large impact parameters of up to $600-1000 \mathrm{~m}$. Joint operation of the first Cherenkov telescopes of the TAIGAIACT and the TAIGA-HiSCORE arrays in the energy range 30-200 TeV will yield a sensitivity of the order of $10^{-13} \mathrm{erg} \mathrm{cm}^{-2} \mathrm{~s}^{-1}$ (for 10 events in 500 hours of observation) and suppress the cosmic ray background by $50-100$ times even for IACT spacing of $1000 \mathrm{~m}$. On its turn, the IACT observing a concrete strong source in the sky, for example the Crab Nebula, can with high efficiency tag the coincident high energy gamma event candidates, which can be used by the TAIGA-HiSCORE array to develop, train and improve its gamma-ray selection methods. The first TAIGA-IACT will be put in operation in autumn 2016.

\section{TAIGA Muon and Tunka-Grande: the nets of particle detectors.}

There are a lot of reasons to include the particle detectors into the hybrid detector complex of the gamma-observatory TAIGA. In particular, the number of muons in a charged cosmic-ray induced EAS is on average 30 times higher than in gamma-ray events [8], so measuring of muon number is very effective way to suppress background. This should work well for the energy range above $100 \mathrm{TeV}$. The overall area of muon detectors $\mathrm{S}_{\mu}$ should be $0.2-0.3 \%$ of the total area of TAIGA-HiSCORE, so we intend to construct a TAIGA-Muon array with an area 2000-3000 $\mathrm{m}^{2}$.

As a first step to future large array to detect EAS electrons and muons we constructed the TunkaGrande array consisting of 19 scintillation stations, each of them with a surface and an underground part. The stations are located at distances about $20 \mathrm{~m}$ from the centres of the Tunka-133 clusters. In November 2015 the deployment of Tunka-Grande was completed and the array was put in operation. Each surface detector includes 12 scintillation counters with a size $80.80 \mathrm{~cm}^{2}$ formerly operated as part of the EAS-TOP and the KASCADE-Grande arrays. There are 8 of the same type counters in underground muon detectors. The simulation results [9] show that for energies $>100 \mathrm{PeV}$ TunkaGrande allows to reconstruct the EAS electron number to $10 \%$ precision, muon number $-25 \%$, the EAS arrival direction to about 1.4 degree accuracy, the core location - $17 \mathrm{~m}$, energy $-20 \%$.

\section{The Tunka-Rex array}

Tunka-Rex is the radio array of the TAIGA observatory [10]. At present time Tunka-Rex consists of 44 radio detectors. They cover area $3 \mathrm{~km}^{2}$ and are located at distance $20 \mathrm{~m}$ about from Tunka-133 cluster centers and scintillation detectors of Tunka-Grande mainly with spacing $200 \mathrm{~m}$. Upon a coincidence trigger of the Cherenkov or the scintillation detectors, both the radio and the airCherenkov or scintillation detector are read out in parallel. The electromagnetic field of the radio signal measured by Tunka-Rex is reconstructed in an effective bandwidth of $35-76 \mathrm{MHz}$. Details on the detector setup and its calibration can be read in reference [11]. There is a clear correlation between the energy and Xmax reconstructed from the radio amplitude measured by Tunka-Rex and the energy reconstructed from the air-Cherenkov light measured by Tunka-133. The Tunka-Rex energy precision seems to be at least as good as the published Tunka-133 resolution of $15 \%$. The Xmax precision of Tunka-Rex is roughly $40 \mathrm{~g} / \mathrm{cm}^{2}$, and can be slightly increased [12]. This resolution is sufficient to statistically distinguish light from heavy primary particles. As a next step, the radio measurements can be combined with the particle detectors to increase the total accuracy for the air-shower parameters: While antennas arrays provide a calorimetric measurement of the electromagnetic component and sensitivity to the longitudinal shower development, measurements of the secondary electrons and muons at ground give a complementary access to the energy and mass composition of the primary particles. In future, this concept can easily be applied to the larger radio array of TAIGA by installing additional antennas. 
XIV International Conference on Topics in Astroparticle and Underground Physics (TAUP 2015) IOP Publishing Journal of Physics: Conference Series 718 (2016) $052006 \quad$ doi:10.1088/1742-6596/718/5/052006

\section{Conclusion}

In 2015, the TAIGA collaboration has continued the construction of a complex of arrays with hybrid detector systems in order to search for new local Galactic sources of gamma rays with energies higher than 20-30 TeV. We will also study signals from the nearby extragalactic sources Mrk421 and Mrk501 in order to investigate the gamma-ray absorption on intergalactic background radiation and to search for axion-photon transitions. The study of gamma radiation in the high energy range is of interest not only for astrophysics, but also for testing theories predicting a violation of Lorenz invariance and to search for super-heavy dark matter. Joint operation of the first Cherenkov telescopes of the TAIGA-IACT and the TAIGA-HiSCORE arrays in the energy range 30-100 TeV will yield a sensitivity of order $10^{-13} \mathrm{erg} \mathrm{cm}^{-2} \mathrm{~s}^{-1}$ (for a $5 \sigma$-detection in 500 hours of observation). This sensitivity would allow to measure the energy spectrum of gamma rays from the Tycho SNRs, a main PeVatron candidate. For TAIGA, placed at $53^{\circ} \mathrm{N} / \mathrm{L}$, this source may be observed during more than 200 hours per year, taking into account $50 \%$ of good weather condition. This sensitivity level would allow us to search for signals from the sources observed by IceCube as neutrinos, if these are of Galactic origin, and would allow to make a survey for new PeVatrons. Figure 4 shows the point source sensitivity of the gamma-observatory TAIGA in comparison with other experiments.

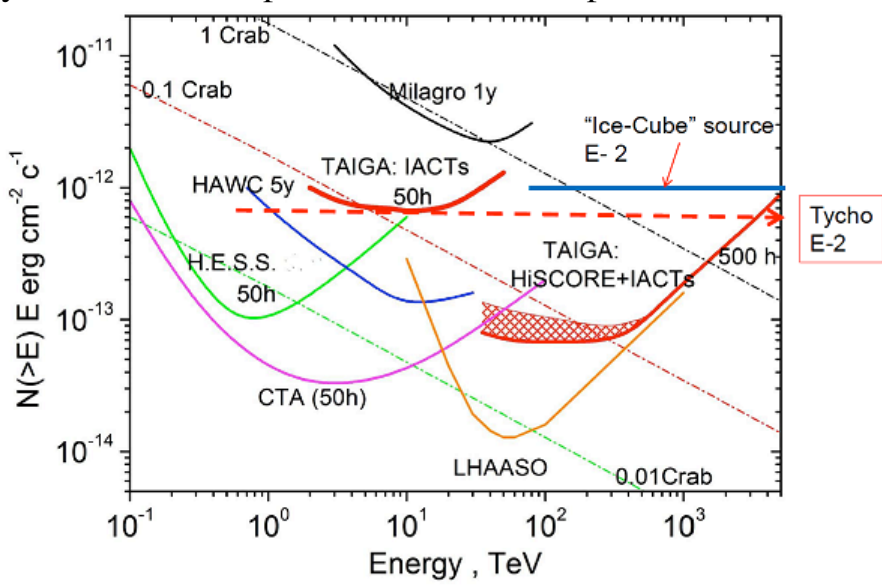

Figure 4. The TAIGA-HiSCORE and TAIGA-IACT point source sensitivity (preliminary) in comparison to other experiments.

\section{Acknowledgments}

This work was supported by the Russian Federation Ministry of Education and Science (agreement 14.B25.31 .0010), the Russian Science Foundation (part 5 and 6, grants 15-12-20022)

\section{References}

[1] Antokhonov B et al 2011 Nucl. Instrum. Meth. A639 42

[2] Berezhnev S et al 2012 Nucl. Instrum. Meth. $\mathbf{A 6 9 2} 98$

[3] Prosin V et al 2014 Nucl. Instrum. Meth. A756 94

[4] Tluczykont et al 2011 Adv. Space Res. 481935

[5] Tluczykont et al 2014 Astropart. Phys. 5642

[6] Gress et al 2013 Nucl. Instrum. Meth. A732 290

[7] Kunnas et al 2014 Nucl. Instrum. Meth. A742 269

[8] Budnev 2014 Journal of Instrumentation 9 C09021

[9] Budnev et al 2014 Moscow University Physics Bulletin 69 Issue 4, 357

[10] Kostunin et al 2014 Nucl. Instrum. Meth. A742 89

[11] Bezyazeekov et al 2015 Nucl. Instrum. Meth. A802 89

[12] Bezyazeekov et al 2015 Preprint arXiv:1509.05652

[13] Wischnewski et al 2015 Proc. 34 ${ }^{\text {th }}$ ICRC, PoS (ICRC2015) 1041 